médecine/sciences $1986 ; 2: 284-5$

La découverte de séquences homologues existant chez lcs mammiferes, notamment la souris et l'homme, avait fait naître l'espoir qu'il pouvait s'agir là d'un fil d'ariane menant à l'identification de gènes contrôlant le développement embryonnaire de ceś espèces. La chose n'était cependant pas évidente et, chez la levure par exemple, de semblables boîtes homéotiques étaient détectées au niveau d'un gène n'ayant rien à voir avec les processus de développement des animaux supérieurs mais plutôt avec le phénomène de détermination sexuelle de la levure [2]. Trois articles parus dans le numéro de novembre de la revue Cell $[3,4,5]$ démontrent qu'en fait les gènes de souris et d'homme au niveau desquels sont retrouvées ces boîtes homéotiques sont homologues entre les deux espèces et qu'ils codent pour de nombreux ARN dont la plupart sont spécifiquement exprimés au cours du développement embryonnaire et(ou) in vitro, au cours de la différenciation de cellules de carcinome embryonnaire. Mieux même, un de ces gènes (ils sont au nombre de ro à 20 , formant plusieurs groupes au niveau desquels ils sont proches les uns des autres sur le même fragment d'ADN) possède une séquence qui ressemble à celle d'un gène homéotique de la drosophile, et pourrait donc exercer une fonction similaire * [6].

Ainsi, grâce aux biologistes et généticiens moléculaires de la drosophile, les embryologistes des mammifères sont-ils maintenant en possession de cet outil qui leur faisait auparavant si cruellement défaut, une sonde permettant d'identifier des gènes jouant un rôle dans le contrôle du développement embryonnaire et de la morphogénèse. En l'absence des mutants de ces gènes qui ont permis d'avancer si vite sur le modèle de la drosophile, peut-être la technique des souris transgéniques permettant d'étudier l'effet de leur hype-

* La fonction du gène en question, le gène "engrailed "est, chez la drosophile, de déterminer la structure des compartiments postérieurs de chaque segment de Tanimal. rexpression ou de leur inactivation par des ARN anti-sens [7] permettra-t-elle de définir leurs fonctions exactes.

A.K.

I. Jarry B, Grau Y. Les gènes du développement. médecine-sciences 1985; 1 : 248-54.

2. Robertson M. Mice, mating types and molecular mechanisms of morphogenesis. Nature 1985; 318: 12-3.

3. Hart CP, Awgulewitsch A, Fainsod A, et al. Homeo-Box Gene Complex on Mouse Chromosome ir: Molecular cloning, expression in Embryogenesis, and Homology to a Human Homeo-Box Locus. Cell I985; 43 : 9-18.
4. Hauser A, Joyner AL, Klein D, et al. Expression of Homologous Homeo-Box Containing Genes in Differentiated Human Teratocarcinoma Cells and Mouse Embryos. Cell 1985; 43 : 19-28. 5. Colberg-Poley AM, Voss SD, Chowdhury K, et al. Clustered Homeo-Boxes are differentially Expressed during Murine Development. Cell 1985; $43: 39-45$.

6. Joyner AL, Kornberg T, Coleman KG, et al. Expression during Embryogenesis of a Mouse Gene with Sequence Homology to the Drosophilia engrailed Gene. Cell 1985; $43: 29-37$.

7. Izant JG, Weintraub $\mathrm{H}$. Constitutive and conditional suppression of exogenous and endogenous genes by antisense RNA. Science $1985 ; 229$ : 345-52.

\title{
AMP cyclique, protéine kinase et contrôles transcriptionnels
}

Les mécanismes de l'action de l'AMP cyclique sur la transcription des gènes eucaryotiques ont été analysés dans un congrès organisé en novembre 1985 par l'Académie des Sciences de Nem York et dans quelques articles récemment publiés. Les résultats les plus nouveaux ont été rapportés par le laboratoire du Pr fungmann à Chicago.

$L ' A M P$ cyclique se fixe normalement aux sous-unités régulatrices des protéines kinases, entraînant leur dissociation d'avec les sous-unités catalytiques ainsi activées. La réaction peut s'écrire $\quad R_{2} \mathrm{C}_{2}+\quad 4 A M P c \rightarrow$ $2 R(A M P C)_{2}+{ }_{2} C[I]$. Il existe plusieurs types de sous-unités régulatrices (probablement trois). La sousunité $R_{\mathrm{II}}$ est phosphorylée par la sous-unité $C$ libérée au cours de la réaction. Le Professeur fungmann et ses collaborateurs [2] ont démontré que la sous-unité $R_{\mathrm{II}}$ purifiée se comportait au niveau de l'ADN comme une "topoisomérase". Cette enzyme a comme propriété de "détordre" l'ADN double brin qui $a$, in vivo, une structure super-enroulée; au cours de cette réaction un brin d'ADN est clivé, désenroulé, et lié à nouveau de façon très spécifique. Les topoisomérases jouent probablement un rôle important chez les microorganismes aussi bien que dans les cellules animales, dans le contrôle de la réplication et de la transcription de l'ADN.

L'activité topoisomérasique de $R_{\mathrm{II}}$ n'est détectable que lorsque la sousunité est phosphorylée et liée à l' $A M P$ cyclique et, de plus, est au moins deux fois plus rapide sur un segment d'ADN contenant un promoteur régulé par l'AMP cyclique que sur de l'ADN tout-venant. Ces résultats suggèrent que l'AMP cyclique pourrait agir en induisant tout d'abord la phosphorylation de $R_{\mathrm{II}}$ par la sous-unité catalytique $C$; le complexe $R_{\mathrm{II}}$ phosphorylé-AMPc serait alors "transloqué " dans le noyau, se fixerait au niveau des promoteurs sensibles à l'AMPc et provoquerait une détorsion locale de l'ADN qui pourrait constituer la base moléculaire de l'effet transcriptionnel.

Ce schéma, quoique attrayant, reste cependant très spéculatif et aurait pour être confirmé à être réconcilié avec d'autres données divergentes. D'une part Nigg et al [3] ont rapporté que le traitement de cellules par 
l' $A M P$ cyclique entraînait la translocation dans le noyau de la seule sous-unité $C, R_{\mathrm{II}}$ restant dans le cytoplasme. Ces résultats furent en fait vivement controversés durant la réunion de Nem York. Plus important, les laboratoires de Richard Hanson (Cleveland, USA) et de Gunther Schütz (Heidelberg, RFA) ont déterminé par différentes méthodes quelle était la séquence d'ADN en amont des gènes de la phosphoenolpyruvate carboxykinase et de la tyrosine aminotransférase qui semblait essentielle à l'action de l'AMP cyclique. Or ce n'est pas préférentiellement à ce niveau que semble se fixer $R_{\mathrm{II}}$. Il n'empêche que la découverte de fungman ouvre une voie nouvelle de recherche, d'autant plus intéres- sante que des résultats similaires ont été présentés récemment par Miguel Beato (Marburg, RFA) concernant l'action du récepteur des glucocorticoïdes liés à l'hormone (Réunion des sociétés allemandes, françaises et suisses de Biochimie, Bâle, octobre I985).

A. $\mathrm{K}$.

I. Munnich A, Vaulont S, Marie J. De nouvelles fonctions pour l'AMP cyclique. médecine-sciences 1985; 1 : $192-17$.

2. Constantinou A L, Squinto SP, Jungmann RA. The phosphoform of the regulatory subunit $R$ II of cyclic AMP-dependent protein kinase possesses intrinsic topoisomerase activity. Cell $1985 ; 42: 429-37$.

3. Nigg EA, Hilz H, Eppenberger HM, Dutly F Rapid and reversible translocation of the catalytic subunit of cAMP-dependent protein kinase type II from the Golgi complex to the nucleus. Embo 7 1985; 4 : 280I-6.

\section{La trans-activation du virus LAV est traductionnel/e}

La synthèse des protéines virales dans une cellule lymphocytaire infectée par le virus LAV responsable du SIDA, est considérablement stimulée par le produit d'un des gènes viraux, le gène Tat III. Le facteur trans-activateur * codé par Tat III reconnaît une courte séquence nucléotidique dénommée TAR (Trans-Activating Responsive sequence) qui est localisée dans le LTR (Long Terminal Repeat) du LAV. Un génome viral délété en gène Tat III synthétise de 500 à 2000 fois plus de produits viraux dans une cellule produisant de façon constitutive (c'est-à-dire permanente) le facteur Tat III que dans

\footnotetext{
* Une activation "en trans " se fait à distance du fragment d $A D N$ qui en est responsable, par opposition à une activation "en cis "qui ne s'exerce que sur un gène contigu au fragment activateur. Un " transactivateur " est un facteur diffusible, produit du gène activateur.

$\mathrm{m} / \mathrm{s} n^{0}$ 5, vol. 2, mai 86
}

une cellule normale. Ce mécanisme de trans-activation également noté pour les virus HTLV et de nombreux virus à $\mathrm{ADN}$, est extrêmement important pour expliquer l'abondante production virale par les cellules infectées et donc en partie le pouvoir pathogène. Rosen et al. du laboratoire de W. Haseltine à Boston (USA), viennent néanmoins de démontrer que les concentrations d'ARN viral, ou de l'ARN d'un gène placé sous le contrôle du LTR du LAV, étaient identiques dans des cellules exprimant ou non le facteur trans-activateur [I].

Afin de déterminer les séquences du LTR viral indispensables au phénomène de trans-activation, les auteurs construisirent plusieurs gènes hybrides comportant, en differentes positions, des fragments du LTR viral. La séquence située entre les bases +38 et +80 (la position $+\mathrm{I}$ définit le site d'initiation de la trans- 